\title{
ORIGINAL ARTICLE Patient participation in care and rehabilitation from the perspective of patients with spinal cord injury
}

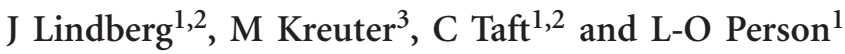

Study design: Qualitative method, semi-structured interviews.

Objectives: The aim of the study was to explore the meaning of patient participation in care and rehabilitation from the perspective of patients with spinal cord injury (SCl).

Setting: Post discharge community setting.

Methods: Semi-structured interviews were performed with 10 persons with SCl representing different ages, gender and levels of injury. All interviews were conducted individually and lasted 40-120 min. The interviews were verbally transcribed and the data were analyzed by means of content analysis.

Results: All informants stressed the importance of patient participation as a necessary prerequisite for successful care and rehabilitation, but emphasized that participation must be tailored to each patient's own preferences, capacities and needs. They also underscored that the staff should be sensitive and responsive to the fact that desired levels and kinds of participation may vary from patient to patient, as well as for the same patient during the course of the rehabilitation. Five themes reflecting central aspects of participation emerged: respect and integrity, planning and decision-making, information and knowledge, motivation and encouragement, and involvement of family.

Conclusions: Patient participation is a critical component of successful SCl rehabilitation and must be facilitated, promoted and tailored to each patient by the staff. Based on the finding from this study a questionnaire has been developed for assessing patient experiences of five domains of participation in rehabilitation to serve as a tool to help in evaluating provided care and in identifying patients' preferences for participation.

Spinal Cord (2013) 51, 834-837; doi:10.1038/sc.2013.97; published online 3 September 2013

Keywords: patient participation; person-centered care; patient-centred care; rehabilitation; spinal cord injury; qualitative research

\section{INTRODUCTION}

Person-centered care (PCC) is a holistic approach that is characterized by respectful and individualized care that empowers patients to actively participate in their care. ${ }^{1}$ The term PCC, as currently used in the rehabilitation field, lacks a consensus definition. However, there appears to be a considerable agreement regarding its core components. ${ }^{2}$ One vital component is patient participation. ${ }^{3}$

Patient participation is considered to be a core element of spinal cord injury (SCI) rehabilitation. ${ }^{4}$ Most SCI rehabilitation units strongly encourage and endorse active patient involvement in care and rehabilitation planning and decision-making. It is based on the theory that an individual's participation in the process is fundamental to its effectiveness. ${ }^{5}$ For instance, it is well known that patients who actively participate in their own care are more adherent to treatments and also report better outcomes and satisfaction with their care. ${ }^{6}$

Even though patient participation is considered desirable and beneficial, little is known about what factors facilitate, promote and contribute to patient participation. However, it is important to recognize that such information should be gleaned directly from the patient. Patients' preferences for participation can differ considerably. ${ }^{7}$ Most patients with SCI want to be as independent as possible, to participate in and be 'in charge' of their rehabilitation. However, they must feel ready and need a supportive relationship with the staff. $^{8}$

The aim of this study was to explore what patient participation in care and rehabilitation means to persons with SCI. Specifically, we were interested in identifying salient domains of patient participation to serve as a base for developing a questionnaire for assessing SCI patients' experiences and preferences of patient participation.

\section{MATERIALS AND METHODS}

Informants

Persons with SCI were strategically selected to represent the SCI population with regard to age, gender and level of injury. Inclusion criteria were: SCI within the past year, discharged from the spinal unit, over 18 years of age, Swedish speaking, no mental illness and treated at a SCI unit in western Sweden. Eligible informants $(n=14)$ were first sent information about the study aims and procedures; 10 persons agreed to participate. They were 24-76 years old; eight of them were men; four were injured in falling accidents and six in traffic accidents; six used a wheelchair and four could walk with supporting aids.

${ }^{1}$ Institute of Health and Care Sciences, Sahlgrenska Academy, University of Gothenburg, Gothenburg, Sweden; ${ }^{2}$ Gothenburg Centre for Person-Centred Care (GPCC), University of Gothenburg, Gothenburg, Sweden and ${ }^{3}$ Institute of Neuroscience and Physiology, Sahlgrenska Academy, University of Gothenburg, Gothenburg, Sweden Correspondence: J Lindberg, Institute of Health and Care Sciences, Sahlgrenska Academy, University of Gothenburg, Box 457 , SE-405 30 Gothenburg, Sweden. E-mail: jeanette.lindberg@gu.se

Received 20 March 2013; revised 9 July 2013; accepted 9 July 2013; published online 3 September 2013 


\section{Procedure}

The interviews lasted between 40 and $120 \mathrm{~min}$ and were conducted individually by one of the authors (L-OP) during the period April-August 2010 . Saturation was reached when 10 informants were interviewed; thus, no further persons were asked to participate.

No definition of 'participation' was given, as we wanted the informants themselves to explain what participation meant for them. The main question asked was: 'What does it mean to you to participate in your own care and rehabilitation'? followed by more specific research questions: 'In which aspects of your care and rehabilitation did you wish to participate'?; 'Do you have any examples of times when you wanted to participate, but were excluded'?; 'Do you have any examples of times when you did not want to participate, but were forced to or persuaded to'?; 'Do you think you had the capacity to fully participate'? and 'Were there any parts of your care and rehabilitation where you did not want to participate'?

\section{Data analysis}

All interviews were tape-recorded and transcribed verbatim. They were analyzed by means of content analysis, a research technique for making replicable and valid inferences from texts to the contexts of their use. ${ }^{9}$ The analysis procedure involved several steps: (1) reading the transcripts through several times to get a sense of wholeness; (2) extracting sentences or phrases with information relevant to the questions (meaning-bearing units); (3) coding meaning-bearing units and grouping them into categories reflecting the core message of the interviews (manifest content) and (4) combining categories into themes (latent content). Two of the authors (L-OP and MK) read all interviews several times and independently extracted preliminary meaningbearing units, categories and themes. One of them suggested more themes than the other one. This was then discussed together with the other authors until a final consensus was reached. A copy of the draft was then sent to the participants to ask them to verify whether their viewpoints were adequately interpreted. ${ }^{10}$

\section{Ethics}

The study was conducted in accordance with the governmental regulations and approved by the Ethical Board of the University of Gothenburg.

\section{RESULTS}

All 10 informants were clearly convinced that patient participation was a necessary prerequisite for successful care and rehabilitation. They were also aware of their own responsibility but stressed the importance the staff has in facilitating and promoting participation. Analyses of the interviews yielded five major themes of participation: respect and integrity, planning and decision-making, information and knowledge, motivation and encouragement, involvement of family member.

\section{Respect and integrity}

Most informants expressed that it was important that the staff get to know them and treat them as individuals and show respect for their personal wishes, preferences and way of being. Several also described situations when staff members were insensitive to their needs for privacy and integrity.

It strengthens you if you have your own wishes and they respect and care about them. And if it works, then it strengthens you mentally as well. (Man, 49 years)

She cut straight to the chase. Came in and asked questions from day one. How long I am up in the evenings, when I get up and things like that. (Man, 31 years)

Taking time to listen was seen as integral to respect. All informants stressed that it was important for the staff to take time to listen to them, for example, by sitting at their bedside and listening to their problems, symptoms and preferences:
Well, it (patient participation) means that they listen to me. They listen and get to know who I am. Then you feel that you participate. (Man, 49 years)

\section{Planning and decision-making}

All informants stressed the importance of being given the opportunity to join in the discussions about their care and rehabilitation, of being asked about their opinions and feelings, of being given the chance to make decisions by themselves, of being informed and given advice about options and of being allowed to choose between those options.

You (the staff) have to explain the options: if you do it like this, then this can happen, and if you do it like that, then this can happen. There are such and such advantages and disadvantages. Then I can choose or I can say that I cannot make that decision, you have to decide. It is then that you are part of the decision making, and I think that is good. (Woman, 77 years)

Some argued that the desire to be actively involved in decisions depended on their health status. When they were newly injured, they felt too weak to actively participate and they preferred to be cared for and let the staff and/or their relatives take responsibility for their care and rehabilitation. When they had regained more vitality and function, the importance of participation increased.

Well, I do not think I was ready to participate until after about a month or two in the hospital. I was so weak that I was just grateful to be cared for. (Woman, 77 years)

\section{Information and knowledge}

Information was regarded as a crucial prerequisite for participation. Most of the informants stressed the need to take responsibility for their care and rehabilitation, but that to do so required that they were well informed. They wanted to know as much as possible about their current situation and prognosis, even when the information or prognosis was negative. It was also considered important that the staff took their time to listen to them and answer their questions.

Yes, I want to have all the information first. I want to know exactly what each pill does, what the care plan is, what they think. So I have asked many questions, that is mainly how I have participated. [...] I have asked about everything: what kind of pills I am taking, why I should take them, what the side effects are, if it is worth taking them. That is how I have taken an interest in my care. (Man, 30 years)

That I get as much information as possible, and in a place where I have an opportunity to ask questions. (Man, 57 years).

\section{Motivation and encouragement}

Several informants stated that it was important that the staff motivate and encourage them to press forward with their training, take the lead in the rehabilitation program and push them toward new goals.

You have to be open [...] listen respectfully to the patient and see what he wants. But at the same time be a step ahead and lead it forward. Because I do not believe that there are any two patients who are alike. (Man, 59 years)

There have to be people who encourage you to train: to get you out of the bed in the [...] when they wake you. (Man, 24 years)

\section{Involvement of family}

Some informants spontaneously talked about the importance of involving their spouses and/or other family members in decisions about their care, particularly in the early phases. They also stated that the SCI has a great impact not only on themselves, but also on their family. 
I think that is good. And I think my wife appreciates it, too. You have another person to discuss things with. [...] at the beginning, it was good that someone else kept track of things. (Man, 59 years)

However, some of the participants were reluctant to involve their family too much. They viewed their rehabilitation to primarily concern themselves and the caring staff. Some also thought that family involvement was not always desirable or beneficial but instead depended on the relationship they had with the family member:

I want to make decisions myself. But I like it when they come and visit. (Man, 72 years)

It depends on how you are and how your relatives are. It can be good and bad. (Man, 30 years)

\section{DISCUSSION}

Patient participation is a core element of $\mathrm{PCC}^{3}$ and the persons with SCI included in this study were clearly convinced that it is a necessary prerequisite for successful SCI rehabilitation. Furthermore, analysis of interviews yielded five central aspects of patient participation, namely being treated with respect by staff; being actively involved in care planning and decision-making; receiving adequate and timely information about care, treatment, condition and prognosis; being motivated and encouraged in rehabilitation; and providing family members' opportunities to be involved. They also stressed that the staff should be sensitive and responsive to the fact that the prominence and desired level of these aspects of participation may vary during the course of SCI treatment and between individual patients, which is in agreement with previous findings. ${ }^{11}$ Many patients prefer to be actively involved in decisions about their care $^{12}$; nonetheless, not all patients want to participate in all aspects of their care and rehabilitation, or to the same extent over time. ${ }^{13,14}$

In our analyses, the theme respect and integrity included two subcategories: 'respect for the person's way of being' and 'respect means listening. The informants felt that the staff should take their time and listen with respect to the patients' needs and preferences. Respect was considered fundamental to creating a relationship in which the patient and staff could freely discuss feelings, options and information. Respect is also commonly emphasized in the PCC and patient participation literature. For example, various studies underline the importance of 'understanding the individual's experience of illness'; 15 or 'respect for values, preferences and expressed needs.'

To facilitate participation in planning and decision-making it is necessary for the staff to recognize the individual's expectations of, capabilities for and/or special obstacles to his/her care and rehabilitation. As has been shown previously, patients with SCI may have different preferences or capacities for participation during the course of rehabilitation and depending on their condition..$^{8,14}$ In our study, this phenomenon was reflected in the subcategory 'active participation is dependent on vitality.' It is important to recognize that there is not only intra-individual variation in patients' preferences for participation but also inter-individual variation. Therefore, patients' preferences for participation vary, and in accordance with a PCC approach, each patient's wish for being involved must be determined and tailored accordingly. ${ }^{7}$ It is important to bear in mind that the patients may prefer to be offered choices and to be asked about their opinions even though they may not always wish to make the final decision. ${ }^{11}$

Information and knowledge are often discussed within the field of PCC, ${ }^{3,15}$ and are possibly the most significant prerequisites for patient participation. ${ }^{16}$ The 'need to know' is a central component in SCI rehabilitation ${ }^{17}$ and 'lack of knowledge' is considered a barrier to coping with SCI. ${ }^{18}$ Progress in rehabilitation hinges on the patient receiving and understanding information about his or her condition. ${ }^{14}$ However, our informants also emphasized that the information should be provided in the right amount, at the right time and in the right place. This means the staff must be sensitive to the individual needs and preferences of the patient and provide information accordingly. Nonetheless, given the immense trauma of a SCI, the ability of the SCI patient to digest and utilize information varies as the patient's situation changes over time and, hence, information should be dispensed gradually. ${ }^{17}$

Motivation and encouragement from the staff were considered essential for patient participation. Motivation is held to be critical in determining outcomes in rehabilitation and is considered important for adjusting to life with SCI. ${ }^{19}$ The informants felt that the staff should encourage them to try new things and motivate them to move forward in their rehabilitation, which in turn was seen to strengthen their self-confidence and sense of responsibility for their rehabilitation. The staff should be sensitive to individual wishes and be a step ahead to push them forward. Staff encouragement may also be viewed as instilling hope in the patient and, as such, may take the form of 'emotional support' 14 and 'need to envision future life possibilities."11

The SCI also affects family members, other close relatives and friends. Involvement of family was stressed in the interviews, and is also emphasized at most SCI units. In line with other studies, ${ }^{17,18}$ we found that the family has an important role in supporting the injured. Nonetheless, family involvement may not always be beneficial. As pointed out by our informants, it is important to acknowledge and respect the individual patient's preferences for family involvement regarding which family members are to be involved and the extent of that involvement.

A strength of this study is that the informants were interviewed within the first year after the injury, thus minimizing recall bias. The retrospective interviews may be considered credible and trustworthy in the sense that they reflect the person's own experiences as they currently experience them, but not necessarily as accurate accounts of the care provided. The credibility of the derived themes was checked by comparing them with findings from similar studies in related fields and in relation to the general theoretical framework of PCC., ${ }^{910}$ Member-checking was also performed in order to increase the creditability of our findings, and did not lead to any significant changes. ${ }^{10}$ The transferability of our results to other contexts is, however, uncertain. The sample was strategically selected to represent the SCI population, but no persons with non-traumatic SCI were included.

Based on the findings from this study a questionnaire for assessing patient experiences of participation in care and rehabilitation has been developed. The questionnaire is aimed to evaluate patient participation and quality of care, as well as to identify patients' preferences for participation. ${ }^{20}$

\section{CONCLUSION}

Patient participation appears to be a necessary and desired prerequisite for successful care and rehabilitation among patients with SCI. Five central aspects of patient participation were identified: respect and integrity, planning and decision-making, information and knowledge, motivation and encouragement, and involvement of family. Moreover, participation in rehabilitation must be tailored to each patient's unique preferences, capacities and needs and the staff should recognize that the extent and ways that patients wish to participate may vary during the course of SCI rehabilitation. 


\section{DATA ARCHIVING}

There were no data to deposit.

\section{CONFLICT OF INTEREST}

The authors declare no conflict of interest.

\section{ACKNOWLEDGEMENTS}

The study was supported by grants from the Gothenburg Centre for PersonCentred Care (GPCC), the Lars Sullivan Memorial Fund for SCI Research, and the Institute of Health and Care Sciences, Sahlgrenska Academy, University of Gothenburg, Sweden.

1 Morgan S, Yoder LH. A concept analysis of person-centered care. J Holist Nurs 2012; 30: 6-15.

2 Leplege A, Gzil F, Cammelli M, Lefeve C, Pachoud B, Ville I. Person-centredness: conceptual and historical perspectives. Disabil Rehabil 2007; 29: 1555-1565.

3 Kitson A, Marshall A, Bassett K, Zeitz K. What are the core elements of patient-centred care? A narrative review and synthesis of the literature from health policy, medicine and nursing. J Adv Nursing 2013; 69: 4-15.

4 Pellatt GC. Patient-professional partnership in spinal cord injury rehabilitation. $\mathrm{Br} \mathrm{J}$ Nurs 2004; 13: 948-953.

5 Kennedy P, Hamilton LR. The needs assessment checklist: a clinical approach to measuring outcome. Spinal Cord 1999; 37: 136-139.

6 Sabaté E. Adherence to Long-Term Therapies: Evidence for Action. World Health Organization: Geneve, 2003.
7 Say R, Murtagh M, Thomson R. Patients' preference for involvement in medical decision making: a narrative review. Patient Educ Coun 2006; 60: 102-114.

8 Angel S, Kirkevold M, Pedersen BD. Rehabilitation after spinal cord injury and the influence of the professional's support (or lack thereof). J Clin Nurs 2011; 20: 1713-1722.

9 Krippendorff K. Content Analysis. An Introduction to its Methodology, 2nd edn Sage Publications, Inc: Thousand Oaks, USA, 2004.

10 Giacomini MK, Cook DJ. Users' guides to the medical literature: XXIII. Qualitative research in health care A. Are the results of the study valid? Evidence-Based Medicine Working Group. JAMA 2000; 284: 357-362.

11 Hammell WK. Experience of rehabilitation following spinal cord injury: a metasynthesis of qualitative findings. Spinal Cord 2007; 45: 260-274.

12 Lund ML, Tamm M, Br nholm IB. Patients' perceptions of their participation in rehabilitation planning and professionals' view of their strategies to encourage it. Occup Ther Int 2001; 8: 151-167.

13 Robinson A, Thomson R. Variability in patient preferences for participating in medical decision making: implication for the use of decision support tools. Qual Health Care 2001; 10 (Suppl 1): 34-i38

14 Sand A, Karlberg I, Kreuter M. Spinal cord injured persons' conceptions of hospital care, rehabilitation, and a new life situation. Scand J Occup Ther 2006; 13: 183-192.

15 Mead N, Bower P. Patient-centredness: a conceptual framework and review of the empirical literature. Soc Sci Med 2000; 51: 1087-1110.

16 Cahill J. Patient participation: a concept analysis. J Adv Nurs 1996; 24: 561-571.

17 May L, Day R, Warren S. Perceptions of patient education in spinal cord injury rehabilitation. Disabil Rehabil 2006; 28: 1041-1049.

18 Babamohamadi H, Negarandeh R, Dehghan-Nayeri N. Barriers to and facilitators of coping with spinal cord injury for Iranian patients: a qualitative study. Nurs Health Sci 2011; 13: 207-215.

19 Trieschmann RB. Spinal Cord Injuries. Psychological, Social and Vocational Rehabilitation, 2nd edn, Demos Publications: New York, 1988.

20 Lindberg J, Kreuter M, Persson L-0, Taft C. Patient Participation in Rehabilitation Questionnaire (PPRQ) —Development and psychometric evaluation. Spinal Cord 2013; 51: 838-842. 